\title{
LIE THEORY OF SEMI-GROUPS OF LINEAR TRANSFORMATIONS
}

\author{
EINAR HILLE
}

1. Introduction. In the Colloquium Lectures which I had the honor of delivering to the Society at the Wellesley meeting in August 1944 , an outline was given of a theory of one-parameter semi-groups of linear bounded operators on a complex $(B)$-space $\mathfrak{X}$ to itself. The problem here is the study of a family of linear bounded transformations $\Im=\{T(\alpha)\}$, defined for $\alpha>0$, with the product law

$$
T(\alpha) T(\beta)=T(\alpha+\beta) .
$$

Such families arise in the most varied branches of classical and of modern analysis and are interesting for their own sake as well as for the many applications.

An extension to the $n$-parameter case was presented to the Society in October 1944 (abstract 51-1-15). Here the parameter $a=\left(\alpha_{1}, \alpha_{2}, \cdots, \alpha_{n}\right)$ is a vector in $n$-dimensional real euclidean space $E_{n}$, the operators $T(a)$ are defined for non-negative values of the components of $a$, and the product law reads

$$
T(a) T(b)=T(a+b)
$$

with $a+b=\left(\alpha_{1}+\beta_{1}, \cdots, \alpha_{n}+\beta_{n}\right)$. These operators commute. If $\|T(a)\|$ is bounded for small $a$, if certain unions of range spaces $T(a)[\mathfrak{X}]$ are dense in the space $\mathfrak{X}$, and if $T(a)$ is a strongly measurable function of $a$, then $T(a)$ is actually strongly continuous for all $a$ and $T(h) x \rightarrow x$ for each $x$ when $h \rightarrow 0$. Further $T(a)$ is the direct product of $n$ commuting one-parameter semi-groups

$$
T(a)=T_{1}\left(\alpha_{1}\right) T_{2}\left(\alpha_{2}\right) \cdots T_{n}\left(\alpha_{n}\right) .
$$

It turned out later that the analysis could be extended, at least in part, to the case in which the parameter set is an open positive cone $\mathbb{E}$ in a $(B)$-space $\mathfrak{B}$. Here $\mathbb{S}$ is an open set, if $a$ and $b$ are in $\sqrt{5}$ so are $\alpha a+\beta b$ for $0 \leqq \alpha, 0 \leqq \beta, 0<\alpha+\beta$. The product law is still given by (1.2).

These investigations with many extensions and numerous applications have now appeared in book form ([6] in the References at the end of this address). The earliest results on continuity in the oneparameter case are due to N. Dunford [2] and extensions to the

The Retiring Presidential address delivered before the Annual Meeting of the Society in Columbus, Ohio, on December 29, 1948; received by the editors December $7,1948$. 
$n$-parameter case have also been found by N. Dunford and I. E. Segal [3]. A number of results referring to the one-parameter case with applications to stochastic processes and Brownian motion have been found independently by $\mathrm{K}$. Yosida whose work is now in course of publication $[10,11,12]$.

So far only commutative operators have been considered and the product law (1.2) is the simplest possible. The non-commutative case has resisted numerous attacks in the past and it is only a few months ago that any headway was made with this problem. I shall have the pleasure of outlining the new theory here; it is a blend of the classical theory of Lie groups with the recent theory of one-parameter semigroups.

2. Assumptions. We shall be concerned with an $n$-parameter family $\subseteq$ of linear bounded operators $T(a)$ on a $(B)$-space $\mathfrak{X}$ to itself. These operators shall form a semi-group, that is, if $T(a)$ and $T(b)$ are in $\mathfrak{S}$ so are their products $T(a) T(b)$ and $T(b) T(a)$ which ordinarily are distinct.

In a first study of the problem, we are entitled to restrict ourselves to comparatively simple situations. Our assumptions will be chosen accordingly, but they will be introduced as needed so as to bring out what parts of the theory require heavy machinery and what may be proved under less restrictive assumptions. No claim is made that the assumptions have their definitive form.

We are dealing with three different spaces, the parameter, the operand, and the operator spaces, and a more or less complicated product law. Consequently we shall need assumptions of diverse nature which may be classified under the following four headings:

(1) The parameter set, that is, the values of $a$ for which $T(a)$ is defined.

(2) The properties of $T(a)$ for fixed $a$.

(3) The properties of $T(a)$ as function of $a$.

(4) The product law, that is, the function

$$
c=F(a, b)
$$

which is defined by the relation

$$
T(c)=T(a) T(b) .
$$

The assumptions relating to (1) and (2) will be kept fixed throughout the following discussion. They are:

$\mathrm{A}^{1} . T(a)$ is defined for $a=\left(\alpha_{1}, \cdots, \alpha_{n}\right)$ in $\bar{E}_{n}^{+}$, that part of $E_{n}$ in which $\alpha_{j} \geqq 0, j=1, \cdots, n$. 
$\mathrm{A}^{2} . T(a)$ is a linear bounded operator on $\mathfrak{X}$ to itself, $T(0)=I$, the identity operator, and $\|T(a)\| \leqq 1$ for $a$ in $\bar{E}_{n}^{+}$.

The initial assumption under (3) is:

$\mathrm{A}_{1}^{3} . T(a)$ is a strongly measurable function of $a$ in $\bar{E}_{n}^{+}$.

The product law will require a number of different assumptions; we start with the following which will be used in the next section. The Euclidean length of the vector $b$ is denoted by $|b|$.

$\mathrm{A}_{1}^{4} . \quad F(a, b)$ is a continuous function on $\bar{E}_{n}^{+} \times \bar{E}_{n}^{+}$to $\bar{E}_{n}^{+}$and

$$
\begin{aligned}
F(a, 0) & =F(0, a)=a, \\
F(a, F(b, c)) & =F(F(a, b), c) .
\end{aligned}
$$

$\mathrm{A}_{2}^{4}$. To every $R>0$ there is a positive $\delta=\delta(R)$ such that $F\left(a, h_{1}\right)$ $\neq F\left(a, h_{2}\right)$ when $h_{1} \neq h_{2}$ provided $|a|<R,\left|h_{1}\right|<\delta,\left|h_{2}\right|<\delta$.

$\mathrm{A}_{3}^{4}$. To every bounded set $K$ whose closure is in $E_{n}^{+}\left(\alpha_{j}>0\right.$, $j=1, \cdots, n)$ there is a positive $\delta=\delta(K)$ such that for $c \in K,|h|<\delta$, the equation $F(h, b)=c$ has a unique solution $b=\psi(c, h)$ in $E_{n}^{+}$which is a continuous function of $(c, h)$ such that for fixed values of $c$ measurable sets correspond to measurable sets.

Some comments are in order at this juncture. We are concerned with a full semi-group and not with a semi-group germ. Assumption $\mathrm{A}^{1}$ is then a natural generalization from one to $n$ dimensions, but it should be realized that a parameter set is admissible if and only if it is closed under the product operation. Thus $\mathrm{A}^{1}$ implies a restriction on the product law and for other choices of $F(a, b)$ we may have to consider other configurations in $E_{n}$ besides $\bar{E}_{n}^{+}$. The complex euclidean space should also be considered. Actually some portions of the theory extend without material change to the case in which the parameter set is the closure of an open positive cone in an arbitrary complex (B)space $\mathfrak{P}$.

The boundedness assumption in $A^{2}$ is very convenient for a first study of the problem. It is a relict, however, of the days when unitary operators in a Hilbert space stood in the foreground and it has the disadvantage of obscuring the fact that the norm of a semi-group operator, while bounded on bounded sets having a positive distance from the boundary of the parameter set, may very well become unbounded when the parameter approaches the boundary. Such questions will have to be relegated to a later study, however.

Assumption $\mathrm{A}_{1}^{3}$ will be discussed in the next section. Condition (2.3) expresses that $T(a) I=I T(a)=T(a)$ and (2.4) implies the associative law $T(a)[T(b) T(c)]=[T(a) T(b)] T(c)$. Assumption $A_{1}^{4}$ alone does not take us anywhere and has to be supplemented by other conditions; $A_{2}^{4}$ and $A_{3}^{4}$ suffice for questions of continuity but for the existence of 
one-parameter sub-semi-groups we shall need Lipschitz conditions and so on.

3. Continuity. In the case of a one-parameter semi-group boundedness of the norm together with strong measurability of the operator function $T(\alpha)$ implies strong continuity of $T(\alpha)$ for $\alpha>0 .{ }^{1}$ It does not imply strong continuity at the origin or continuity in the uniform operator topology for $\alpha>0$. This result extends to the present situation.

THEOREM 3.1. Under the assumptions of $\$ 2$, omitting $\mathrm{A}_{2}^{4}$, the operator $T(a)$ is strongly continuous in $E_{n}^{+}$, that is, for $\alpha_{j}>0, j=1, \cdots, n$.

The proof uses the same principles as in the one-dimensional case, that is, the product law plus continuity of a definite integral with respect to translations. Let $D$ be a bounded domain the closure of which is in $E_{n}^{+}$. By virtue of $A_{3}^{4}$ there exists a positive $\eta=\eta(D)$ such that for each $c$ in $D$ and each $a=\left(\alpha_{1}, \cdots, \alpha_{n}\right)$ with $0 \leqq \alpha_{j} \leqq \eta$, $j=1, \cdots, n$, the equation

$$
c=F(a, b)
$$

has a unique solution

$$
b=\psi(c, a)
$$

which is a continuous function of $(c, a)$ in the product set in question. In particular, $\left|\psi\left(c_{1}, a\right)-\psi\left(c_{2}, a\right)\right|$ is small when $\left|c_{1}-c_{2}\right|$ is small and this holds uniformly in $a$.

For $c_{1}$ and $c_{2}$ in $D$ we have then

$$
T\left(c_{1}\right) x-T\left(c_{2}\right) x=T(a)\left[T\left(\psi\left(c_{1}, a\right)\right) x-T\left(\psi\left(c_{2}, a\right)\right) x\right] .
$$

The right side being independent of $a$, we may integrate the identity with respect to $a$ over the cube $C(\eta), \frac{1}{2} \eta \leqq \alpha_{j} \leqq \eta, j=1, \cdots, n$, obtaining

$$
\left(\frac{1}{2} \eta\right)^{n}\left[T\left(c_{1}\right) x-T\left(c_{2}\right) x\right]=\int_{C(\eta)} T(a)\left[T\left(\psi\left(c_{1}, a\right)\right) x-T\left(\psi\left(c_{2}, a\right)\right) x\right] d a
$$

whence

$$
\left(\frac{1}{2} \eta\right)^{n}\left\|T\left(c_{1}\right) x-T\left(c_{2}\right) x\right\| \leqq \int_{C(\eta)}\left\|T\left(\psi\left(c_{1}, a\right)\right) x-T\left(\psi\left(c_{2}, a\right)\right) x\right\| d a .
$$

\footnotetext{
${ }^{1}$ R. S. Phillips has recently shown that the boundedness assumption is superfluous and strong measurability alone is necessary and sufficient for strong continuity. [Added in proof March, 1950.]
} 
Since $T(b)$ is a strongly measurable function of $b$ and in the correspondence $b=\psi(c, a)$ measurable $b$-sets are the images of measurable $a$-sets, the operator $T(\psi(c, a))$ is strongly measurable in $a$, so that the integrand is a bounded Lebesgue measurable function of $a$. From the fact that $\psi\left(c_{2}, a\right) \rightarrow \psi\left(c_{1}, a\right)$ when $c_{2} \rightarrow c_{1}$, the convergence being uniform with respect to $a$ in $C(\eta)$, one may infer that the integral tends to zero when $c_{2} \rightarrow c_{1}$. It follows that $T(c)$ is strongly continuous at $c=c_{1}$ and hence everywhere in $E_{n}^{+}$.

Ordinarily we cannot prove continuity in the uniform topology, but if we assume uniform measurability at the outset, then $x$ may be suppressed everywhere in the proof and uniform continuity results.

Assumption $\mathrm{A}^{2}$ is evidently used twice in the proof, but in both places it could be replaced by the weaker assumption that $\|T(a)\|$ is bounded in every bounded domain whose closure lies in $E_{n}^{+}$. In the case of a separable space $\mathfrak{X}$, weak measurability implies the strong kind so that "strongly" could be replaced by "weakly" in $A_{1}^{3}$. In this case it is also likely that we may dispense with the boundedness condition on the norm altogether, merely assuming $\|T(a)\|$ to be finite in $E_{n}^{+}$. This is suggested by the following considerations.

If $\mathfrak{X}$ is separable and $T(a)$ is weakly measurable in $E_{n}^{+}$, then $\log \|T(a)\|$ is measurable Lebesgue and different from $+\infty$. Further it satisfies the inequality

$$
f(c) \leqq f(a)+f(b), \quad c=F(a, b),
$$

which is the proper generalization of the subadditive inequality

$$
f(a+b) \leqq f(a)+f(b)
$$

corresponding to the case $F(a, b)=a+b$. For the latter it is known that a solution, defined in $E_{n}^{+}$, which is measurable and different from $+\infty$, is bounded above in every bounded interior domain $D$ whose closure lies in $E_{n}^{+}$(see [6, p. 135] for the case $n=1$, the extension to arbitrary $n$ has been given by R. A. Rosenbaum). In principle the method of the proof also extends to (3.3) but the analytical and topological difficulties are considerable so the discussion of this question has to be postponed to another occasion.

One can get fairly trivial examples indicating that $T(a)$ need not be continuous on the boundary of $E_{n}^{+}$under the assumptions of Theorem 3.1, not even in the weak sense. ${ }^{2}$ In particular, continuity is apt to fail at the origin. A common cause of such failure is that the union of the range spaces, $\bigcup_{a} T(a)[\mathfrak{X}]$, is not dense in $\mathfrak{X}$. In the one-

\footnotetext{
${ }^{2}$ In the one-parameter case it may be shown that weak continuity to the right at the origin implies strong continuity. [Added in proof.]
} 
parameter case it would be sufficient to add the assumption that $\mathrm{U}_{\alpha} T(\alpha)[\mathfrak{X}]$ is dense in $\mathfrak{X}$ in order to obtain right-hand continuity at $\alpha=0$. It is not clear at the time of writing this if such a condition would suffice as additional assumption to ensure continuity of $T(a)$ on the boundary of $E_{n}^{+}$. On the other hand, if $n=1$ the assumption that $T(\alpha) x \rightarrow x$ for each $x$ when $\alpha \rightarrow 0$ suffices for strong continuity in $\bar{E}_{1}^{+}$and this result extends to the general case. We assume

$\mathrm{A}_{2}^{3} . T(a)$ is strongly continuous at $a=0$.

Theorem 3.2. If $T(a)$ satisfies $\mathrm{A}_{2}^{3}$ and the conditions of $\$ 2$, except $\mathrm{A}_{1}^{3}$ and $\mathrm{A}_{3}^{4}$, then $T(a)$ is strongly continuous in $\bar{E}_{n}^{+}$.

If $x$ and $\epsilon>0$ are given, we can find an $\eta$ so small that $\|T(h) x-x\|$ $<\epsilon$ for $h \in S(0)=E\left(|h|<\eta, h \in \bar{E}_{n}^{+}\right)$. The mapping

$$
T_{a}: \quad h \rightarrow F(a, h), \quad a \in \bar{E}_{n}^{+},
$$

takes $S(0)$ into a set $S(a)$. If $\left|a_{0}\right|<R$ and $\eta<\delta(R)$, as we may assume, condition $\mathrm{A}_{2}^{4}$ asserts that the mapping $T_{a_{0}}$ is one-to-one so that $S\left(a_{0}\right)$ is an $n$-cell and Int $\left[S\left(a_{0}\right)\right] \neq \varnothing$. Thus $S\left(a_{0}\right)$ contains a sphere of center $c_{0}$ and radius $\rho$, say. But $F(a, h)$ is continuous by $\mathrm{A}_{1}^{4}$ so there exists a $\delta_{0}$ such that $\left|F(a, h)-F\left(a_{0}, h_{0}\right)\right|<\rho$ if $\left|a-a_{0}\right|<\delta_{0},\left|h-h_{0}\right|$ $<\delta_{0}$ where $h, h_{0} \in S(0)$. Here we choose $h_{0}$ so that $c_{0}=F\left(a_{0}, h_{0}\right)$. We can then be sure that $S(a) \cap S\left(a_{0}\right) \neq \varnothing$ if $\left|a-a_{0}\right|<\delta_{0}$. Hence if $c=F\left(a, h_{1}\right)=F\left(a_{0}, h_{2}\right)$ is a common point of $S(a)$ and $S\left(a_{0}\right)$ we have

$$
\begin{aligned}
\left\|T(a) x-T\left(a_{0}\right) x\right\| & \leqq\|T(c) x-T(a) x\|+\left\|T(c) x-T\left(a_{0}\right) x\right\| \\
& =\left\|T(a)\left[T\left(h_{1}\right) x-x\right]\right\|+\left\|T\left(a_{0}\right)\left[T\left(h_{2}\right) x-x\right]\right\| \\
& \leqq\left\|T\left(h_{1}\right) x-x\right\|+\left\|T\left(h_{2}\right) x-x\right\| \leqq 2 \epsilon
\end{aligned}
$$

where $\left|a-a_{0}\right|<\delta_{0}$. This proves that $T(a) x$ is continuous at $a=a_{0}$. Hence $T(a)$ is strongly continuous in $\bar{E}_{n}^{+}$.

4. A functional equation. In the study of one-parameter sub-semigroups we encounter the functional equation

$$
g(\rho+\sigma)=F[g(\rho), g(\sigma)]
$$

where $g(\rho)$ is a function on positive numbers to $\bar{E}_{n}^{+}$. For this problem we need further information concerning $F(a, b)$.

$\mathrm{A}_{4}^{4}$. There exists a fixed positive constant $B$ such that for all points in $\bar{E}_{n}^{+}$we have

$$
\begin{aligned}
& \left|F\left(a_{1}, b\right)-F\left(a_{2}, b\right)\right| \leqq[1+B|b|]\left|a_{1}-a_{2}\right| \\
& \left|F\left(a, b_{1}\right)-F\left(a, b_{2}\right)\right| \leqq[1+B|a|]\left|b_{1}-b_{2}\right| .
\end{aligned}
$$


$\mathrm{A}_{5}^{4}$. There exists a positive monotone increasing continuous function $\omega(\xi), 0<\xi<\infty$, which tends to zero with $\xi$, such that

$$
\begin{gathered}
F(a, b)=a+b+G(a, b), \\
|G(a, b)| \leqq r \omega[|a+b|], \quad r=\min (|a|,|b|) .
\end{gathered}
$$

It would be possible to combine these inequalities in such a manner that they refer to the behavior of $\left|F\left(a, b_{1}\right)-F\left(a, b_{2}\right)-b_{1}+b_{2}\right|$ and $\left|F\left(a_{1}, b\right)-F\left(a_{2}, b\right)-a_{1}+a_{2}\right|$. Such inequalities are basic in the study of analytical group germs in a $(B)$-space due to $\mathrm{G}$. Birkhoff [1]. Our conditions seem to be slightly better adapted to the needs of the methods used below. They are also closely related to the assumptions of P. A. Smith [8, 9]. See further I. E. Segal [7] whose work suggests that the Lipschitz condition might be inessential but it is not clear to me at the moment how his methods could be brought to bear on the present problem. In making comparisons the reader should keep in mind that we are dealing with a fixed coordinate system, a semi-group rather than a group, and a situation in the large. All the results of the present section hold for the case in which $E_{n}$ is replaced by an arbitrary $(B)$-space and $\bar{E}_{n}^{+}$by the closure of an open positive cone.

With an arbitrary element $b \in \bar{E}_{n}^{+}$we also consider its successive "powers" defined by

$$
b^{(1)}=b, b^{(m)}=F\left(b, b^{(m-1)}\right) .
$$

When $b$ and $m$ are bulky expressions we shall write $(b ; m)$ for $b^{(m)}$ A basic property of the powers is given in the following

LemmA. Let $\tau$ be defined by $\omega(\tau)=\frac{1}{2}$. If $b \in \bar{E}_{n}^{+}$and $m$ is a positive integer such that $m|b| \leqq \frac{1}{2} \tau$, then

$$
b^{(m)}=m b+R_{m}(b), \quad\left|R_{m}(b)\right| \leqq m|b| \omega(2 m|b|) .
$$

The lemma holds for $m=2$ by $\mathrm{A}_{5}^{4}$ and is proved by induction using the inequality

$$
\left|R_{m+1}(b)\right| \leqq\left|R_{m}(b)\right|+|b| \omega\left[(k+1)|b|+\left|R_{m}(b)\right|\right] .
$$

We can now state and prove

THEOREM 4.1. Under the assumptions $\mathrm{A}_{1}^{4}, \mathrm{~A}_{4}^{4}$, and $\mathrm{A}_{5}^{4}$ the equation (4.1) with the initial condition

$$
\lim _{\rho \rightarrow 0} \rho^{-1} g(\rho)=a \in \bar{E}_{n}^{+}
$$

has a unique solution $g(\rho)=f(\rho a)$ in $\bar{E}_{n}^{+}$. The solution is an absolutely 
continuous function of $\rho$ with bounded derived numbers and for $0 \leqq \rho \leqq \sigma$

$$
|g(\sigma)-g(\rho)| \leqq B^{-1}\left[e^{B \sigma|a|}-e^{B \rho|a|}\right] \text {. }
$$

Starting with an arbitrary element $b$ of $\bar{E}_{n}^{+}$we form a sequence of powers $(b / \nu)^{(\mu)}=(b / \nu ; \mu)$ where $\mu$ and $\nu$ are positive integers. Using $\mathrm{A}_{4}^{4}$ and induction on $\mu$ one shows that

$$
|(b / \nu ; \mu+1)-(b / \nu ; \mu)| \leqq \frac{|b|}{\nu}\left\{1+\frac{B|b|}{\nu}\right\}^{\mu},
$$

whence we obtain

$$
|(b / \nu ; \mu)| \leqq B^{-1}\left[e^{(\mu / \nu) B|b|}-1\right]
$$

for all $\mu, \nu$.

In the following $\mu=\nu$ will be a power of 2 and we shall investigate the convergence of the sequence $\left\{\left(2^{-i} b ; 2^{j}\right)\right\}$. Here $\left(2^{-i} b ; 2^{j}\right)$ is obviously the square of $\left(2^{-i b} ; 2^{j-1}\right)$. Using this fact, $(4.8)$, and $\mathrm{A}_{4}^{4}$ repeatedly, we obtain for $j<k$

$$
\begin{aligned}
\Delta_{j, k}(b) & =\left|\left(2^{-k} b ; 2^{k}\right)-\left(2^{-j} b ; 2^{j}\right)\right| \\
& \leqq 2^{j} e^{B|b|}\left|\left(2^{-k} b ; 2^{k-j}\right)-2^{-j} b\right| .
\end{aligned}
$$

Let $R$ be a fixed positive number, arbitrarily large, and restrict $b$ to the sphere $|b| \leqq R$. Suppose that $j$ is so large that $2^{1-j} R \leqq \tau$. We can then apply the lemma with $b$ replaced by $2^{-k} b$ and $m$ by $2^{k-i}$. After some simplification we obtain

$$
\Delta_{j, k}(b) \leqq|b| e^{B|b|} \omega\left(2^{1-j}|b|\right) .
$$

It follows that the sequence $\left\{\left(2^{-i} b ; 2^{j}\right)\right\}$ converges to a limit and we set

$$
f(b)=\lim _{j \rightarrow \infty}\left(2^{-j} b ; 2^{j}\right) .
$$

The convergence being uniform with respect to $b$ for $|b| \leqq R$, we conclude that $f(b)$ is a continuous function of $b$.

In particular the limit exists for $b=\rho a$, uniformly with respect to $\rho$ for $0 \leqq \rho \leqq R<\infty$ so that $f(\rho a)$ is a continuous function of $\rho$.

From (4.10) we conclude also that

$$
f(b ; m) \equiv \lim _{j \rightarrow \infty}\left(2^{-i b} ; m 2^{j}\right)
$$

exists and equals the $m$ th power of $f(b)$. But this implies that

$$
\lim _{j \rightarrow \infty}\left(2^{-i} m^{-1} b ; m 2^{i}\right)=f\left(b m^{-1} ; m\right)
$$


exists. We want to show that the latter limit is actually independent of $m$ and consequently equals $f(b ; 1)=f(b)$. For this purpose we consider

$$
\Delta_{k, m}(b, j)=\left|\left(2^{-i} k^{-1} b ; k 2^{j}\right)-\left(2^{-i} m^{-1} b ; m 2^{j}\right)\right| .
$$

Here we use the fact that the terms on the right are squares together with $\mathrm{A}_{4}^{4}$ and (4.8) to obtain the estimate

$$
\begin{aligned}
\Delta_{k, m}(b, j) & \leqq 2 e^{\frac{1}{B}|b|} \Delta_{k, m}\left(2^{-1} b, j-1\right) \leqq \cdots \\
& \leqq 2^{i} e^{B|b|} \Delta_{k, m}\left(2^{-i} b, 0\right) .
\end{aligned}
$$

If $2^{1-i}|b| \leqq \tau$, we can apply the lemma once more and see that

$$
2^{i} \Delta_{k, m}\left(2^{-i} b, 0\right)<2 \omega\left(2^{1-i}|b|\right) \rightarrow 0
$$

when $j \rightarrow \infty$. It follows that

$$
f\left(b k^{-1} ; k\right)=f\left(b m^{-1} ; m\right)=f(b ; 1)=f(b)
$$

as asserted. Hence with $b=k a$ we have $f(a ; k)=f(k a)$ for every positive integer $k$.

From this it follows that $f(\rho a)$ satisfies (4.1), to start with for positive integral values of $\rho$ and $\sigma$ which implies that it also holds for rational values and finally, $f(\rho a)$ being continuous, for all positive real values.

The argument used for the convergence proof also gives the inequality

$$
\begin{aligned}
\left|\left(2^{-k} b ; 2^{k}\right)-\left(2^{-k} c ; 2^{k}\right)\right| & \leqq 2^{k} e^{B|c|}\left|2^{-k} b-2^{-k} c\right| \\
& =e^{B|c|}|b-c|
\end{aligned}
$$

if $|b| \leqq|c|$. Passing to the limit with $k$ we obtain the Lipschitz condition

$$
|f(b)-f(c)| \leqq e^{B|c|}|b-c|, \quad|b| \leqq|c| .
$$

It follows that $f(\rho a)$ satisfies a Lipschitz condition with respect to $\rho$. Formula (4.7) is an immediate consequence of (2.3), (4.1), $\mathrm{A}_{4}^{4}$, and the estimate

$$
|f(b)| \leqq B^{-1}\left[e^{B|b|}-1\right]
$$

which follows from (4.8).

In order to verify the initial condition we revert to the lemma once more. We have

$$
\left(2^{-i} \rho a ; 2^{j}\right)=\rho a+R_{2^{i}}\left(2^{-i} \rho a\right)
$$


and if $2 \rho|a| \leqq \tau$ the norm of the remainder does not exceed $\rho|a| \omega(2 \rho|a|)$. When $j$ tends to infinity, the left member tends to $f(\rho a)$. It follows that

$$
|f(\rho a)-\rho a| \leqq \rho|a| \omega(2 \rho|a|)
$$

for $2 \rho|a| \leqq \tau$ so the initial condition is satisfied.

For the uniqueness proof we shall need only $A_{4}^{4}$. Suppose that $g(\rho)$ and $h(\rho)$ are two solutions of (4.1) and (4.6) in $\bar{E}_{n}^{+}$. We may assume that they both satisfy (4.13) with $b$ replaced by $\rho a$; if necessary we restrict $\rho$ to a fixed finite interval and replace $B$ by a larger constant. We have then by the usual square root argument

$$
\begin{aligned}
|g(\rho)-h(\rho)| & =\left|F\left[g\left(\frac{1}{2} \rho\right), g\left(\frac{1}{2} \rho\right)\right]-F\left[h\left(\frac{1}{2} \rho\right), h\left(\frac{1}{2} \rho\right)\right]\right| \\
& \leqq 2 e^{\frac{1}{3} B_{\rho}|a|}\left|g\left(\frac{1}{2} \rho\right)-h\left(\frac{1}{2} \rho\right)\right|,
\end{aligned}
$$

whence by iteration

$$
|g(\rho)-h(\rho)| \leqq 2^{k} e^{B \rho|a|}\left|g\left(2^{-k} \rho\right)-h\left(2^{-k} \rho\right)\right|
$$

and this tends to zero when $k \rightarrow \infty$ by virtue of the common initial condition. Hence $g(\rho) \equiv h(\rho)$ and our sketch of the proof of Theorem 4.1 is complete.

This theorem may be strengthened in a direction which will be useful below.

THEOREM 4.2. If $\omega(\xi)$ satisfies the integrability condition

$$
\int_{0}^{1} \xi^{-1} \omega(\xi) d \xi<\infty
$$

and if $g(\rho)$ is a solution of (4.1) in $\bar{E}_{n}^{+}$which tends to zero with $\rho$, then there exists an a in $\bar{E}_{n}^{+}$such that (4.6) holds.

Suppose that $\rho_{0}$ is so small that $2|g(\rho)| \leqq \tau$ for $\rho \leqq \rho_{0}$. Repeated use of $\mathrm{A}_{5}^{4}$ gives

$$
\begin{aligned}
g(\rho) & =2^{k} g\left(2^{-k} \rho\right)+R_{k}(\rho), \\
\left|R_{k}(\rho)\right| & \leqq \sum_{\nu=1}^{k} 2^{\nu-1}\left|g\left(2^{-\nu} \rho\right)\right| \omega\left[2\left|g\left(2^{-\nu} \rho\right)\right|\right] .
\end{aligned}
$$

On the other hand

$$
\begin{aligned}
|g(\rho)| & \geqq\left\{1-\frac{1}{2} \omega\left[2\left|g\left(2^{-1} \rho\right)\right|\right]\right\} 2\left|g\left(2^{-1} \rho\right)\right| \geqq \cdots \\
& \geqq \prod_{j=1}^{\nu}\left\{1-\frac{1}{2} \omega\left[2\left|g\left(2^{-i} \rho\right)\right|\right]\right\} 2^{\nu}\left|g\left(2^{-\nu} \rho\right)\right| .
\end{aligned}
$$


It follows that

$$
\left|R_{k}(\rho)\right| \leqq \frac{1}{2}|g(\rho)| \sum_{\nu=1}^{k} \prod_{j=1}^{\nu}\left\{1-\frac{1}{2} \omega\left[2\left|g\left(2^{-j} \rho\right)\right|\right]\right\}^{-1} \omega\left[2\left|g\left(2^{-\nu} \rho\right)\right|\right] .
$$

Here the right-hand member tends to a finite limit when $k \rightarrow \infty$ if and only if

$$
\sum_{\nu=1}^{\infty} \omega\left[2\left|g\left(2^{-\nu} \rho\right)\right|\right]<\sum_{\nu=1}^{\infty} \omega\left[\left(\frac{2}{3}\right)^{\nu} \tau\right]
$$

is convergent where the inequality is a trivial consequence of (4.16). But the second series converges by virtue of (4.15). It follows that $R_{k}(\rho)$ converges uniformly to a continuous limit for $0 \leqq \rho \leqq \rho_{0}$ when $k \rightarrow \infty$ and this implies that

$$
\lim _{k \rightarrow \infty} 2^{k} g\left(2^{-k} \rho\right) \equiv l(\rho)
$$

exists as a continuous function of $\rho$. Furthermore, one sees that if $l(\rho)=\rho m(\rho)$ then $m(2 \rho)=m(\rho)$.

In exactly the same manner one proves that

$$
\lim _{k \rightarrow \infty} 3^{k} g\left(3^{-k} \rho\right)
$$

exists and a more detailed analysis shows that it also equals $l(\rho)$. This forces $m(\rho)$ to have the additional property $m(3 \rho)=m(\rho)$, and $\log 2$ and $\log 3$ being incommensurable, this makes the continuous function $m(\rho)$ equal to a constant, $a$ say. Since $g(\rho) \in \bar{E}_{n}^{+}$by assumption, $a$ will have the same property. From $l(\rho)=\rho a$, we conclude that (4.6) holds.

Condition (4.15) is not particularly restrictive and it is fully utilized in the proof of the theorem, but we have of course no evidence whatsoever that it is a necessary condition.

5. The one-parameter sub-semi-groups. In view of the discussion in $\S 3$, it is natural to replace $A_{1}^{3}$ by

$\mathrm{A}_{3}^{\mathbf{3}}$. $T(a)$ is a strongly continuous function of $a$ in $\bar{E}_{n}^{+}$.

The basic theorem on sub-semi-groups reads

THEOREM 5.1. Let the semi-group $\subseteq=\{T(a)\}$ satisfy assumptions $\mathrm{A}^{1}, \mathrm{~A}^{2}, \mathrm{~A}_{3}^{3}, \mathrm{~A}_{1}^{4}, \mathrm{~A}_{4}^{4}$, and $\mathrm{A}_{5}^{4}$. If $a$ is any element of $\bar{E}_{n}^{+}$, then the sequence of operators $\left\{\left[T\left(2^{-j} a\right)\right]^{2^{j}}\right\}$ converges strongly to a limit $S(a)=T[f(a)]$ where $f(a)$ is defined by (4.10). The operators $\{S(\rho a)\}, 0<\rho<\infty$, form a sub-semi-group $\mathfrak{S}_{a}$ of $\mathfrak{S}$ and $\mathfrak{S}_{\alpha a}=\mathfrak{S}_{a}$ if $\alpha>0$. If (4.15) also holds, then conversely if $\mathfrak{T}=\{S(\rho)\}, 0<\rho<\infty$, is a sub-semi-group of $\mathfrak{S}$, if 
$S(\rho)=T[g(\rho)]$ and $\lim _{\rho \rightarrow 0} g(\rho)=0$, then there exists an $a \in \bar{E}_{n}^{+}$such that $\mathfrak{T}=\mathfrak{\Im}_{a}$.

The proof follows directly from Theorem 4.1 and 4.2. Referring back to $A_{1}^{4}$ and the definition of the power $\left(2^{-j} a ; 2^{j}\right)$ one sees that

$$
\left[T\left(2^{-i} a\right)\right]^{2^{j}}=T\left[\left(2^{-j} a ; 2^{j}\right)\right] \text {. }
$$

By (4.10) we know that $\left(2^{-j} a ; 2^{j}\right) \rightarrow f(a)$ when $j \rightarrow \infty$ and by $A_{3}^{3}$

$$
T\left[\left(2^{-i} a ; 2^{j}\right)\right] x \rightarrow T[f(a)] x \equiv S(a) x
$$

for each $x$. That the operators $S(\rho a), 0<\rho<\infty$, form a semi-group with the product law $S(\rho a) S(\sigma a)=S((\rho+\sigma) a)$ follows from the fact that $f(\rho a)$ satisfies equation (4.1).

If (4.15) holds, then we can apply Theorem 4.2. If then $\mathfrak{T}=\{S(\rho)\}$ is a one-parameter sub-semi-group of $\subseteq$ with the canonical product law, we can find a function $g(\rho)$ satisfying (4.1) such that $S(\rho)$ $=T[g(\rho)]$. By assumption $g(\rho) \rightarrow 0$ with $\rho$ so there is an $a$ in $\bar{E}_{n}^{+}$such that $g(\rho)=f(\rho a)$ and, hence, $\mathfrak{T}=\mathfrak{S}_{a}$.

The equation

$$
p=f(\rho a)
$$$$
0<\rho<\infty
$$

defines a path $\Gamma_{a}$ in $\bar{E}_{n}^{+}$, starting at the origin where it is tangent to the vector $p=\rho a$. The properties of $f(\rho a)$ listed in Theorem 4.1 show that $\Gamma_{a}$ has a tangent almost everywhere and every arc of $\Gamma_{a}$ corresponding to $0<\rho<R$ is rectifiable. If $F(p, q)$ has continuous partial derivatives with respect to the components of $q$, we may show that $f(\rho a)=\left[\phi_{1}(\rho), \cdots, \phi_{n}(\rho)\right]$ is a solution of the system of first order differential equations

$$
\left\{\begin{array}{l}
\frac{d \phi_{j}}{d \rho}=\sum_{k=1}^{n} \alpha_{k} F_{j, k}\left(\phi_{1}, \cdots, \phi_{n} ; 0, \cdots, 0\right), \quad i=1, \cdots, n, \\
\phi_{j}(0)=0 .
\end{array}\right.
$$

Here $F_{j, k}$ is the derivative of the $j$ th component of $F(p, q)$ with respect to the $k$ th component of $q$ and $a=\left(\alpha_{1}, \cdots, \alpha_{n}\right)$. This system, when available, usually offers more convenient determination of $\Gamma_{a}$ than the functional equation (4.1) with the initial condition (4.6). It should be observed, however, that the latter define $\Gamma_{a}$ uniquely in situations where the uniqueness theorems for differential equations do not apply and even the differential equations themselves may fail to exist.

Every path $\Gamma_{a}$ is confined to $\bar{E}_{n}^{+}$and condition (4.15) may be used to show that $\Gamma_{a}$ cannot return to the origin when $\rho$ tends to a finite limit 
or to infinity. Under the same assumption, $\Gamma_{a}$ is a simple arc. The general question of what happens to $f(\rho a)$ when $\rho \rightarrow \infty$ is very important. A particularly simple case is that in which $f(\rho a)$ tends to a finite limit $p_{0}$ for then $p_{0}=F\left(p_{0}, p_{0}\right)$ and $T\left(p_{0}\right)$ is a projection operator.

A transformation semi-group, in contrast to a group, may contain projections and their parameters are determined by the equation

$$
F(p, p)=p
$$

which defines a locus $P$ in $\bar{E}_{n}^{+} . P$ always contains $p=0$ and may reduce to this point. The origin is an isolated point of $P$ since $|F(p, p)-p|$ $>0$ as long as $p \neq 0$ and $\omega(2|p|)<1$ as we see from $A_{5}^{4}$. If a path $\Gamma_{a}$ has an interior point $p_{0}=f(\omega a)$ in common with $P$, then the operator $T[f(\rho a)]$ is periodic with period $\omega$ for $\rho>\omega$. This cannot happen, however, if condition (4.15) holds and if Int $\Gamma_{a} \epsilon E_{n}$. A path $\Gamma_{a}$ may very well have its end point on $P$ and $P$ may be made up of such terminal points. This happens in the case of the projective semi-group on positive numbers for which all paths $\Gamma_{a}$ are straight line segments joining the origin with the surface $P$ which is a portion of a hyperbolic paraboloid in $E_{3}$.

The determination of all points $p$ such that $p=f(a)$ for some $a$ appears to be very difficult. It is clear that if $p=f(a)$, then we must be able to determine a sequence of points $\left\{p_{k}\right\}$ in $\bar{E}_{n}^{+}$such that $p_{0}=p$ and

$$
F\left(p_{k}, p_{k}\right)=p_{k-1}, \quad k=1,2,3, \cdots .
$$

Since $p_{k}=f\left(2^{-k} a\right)$ we must also have

$$
\lim _{x \rightarrow \infty} 2^{k} p_{k}=a \text {. }
$$

It is possible to determine conditions under which this process may be carried through, but so far the results have been rather disappointing. In our theory it is much easier to determine the paths from the origin than to find the path, if any, which joins a given point $p$ with the origin.

Let us observe that if $p=f(a)$ then

$$
T(p) x=\lim _{\delta \rightarrow 0} \exp \left\{\frac{1}{\delta}[T(f(\delta a))-1]\right\} x, \quad x \in \mathfrak{X},
$$

in the sense of strong convergence. Cf. [6, p. 189].

6. The infinitesimal generators. With each one-parameter subsemi-group $\mathfrak{S}_{a}$ defined above there is associated an infinitesimal gen- 
erator $A(a)$ of $\mathfrak{S}$ which is defined by

$$
\lim _{\delta \rightarrow 0} \frac{1}{\delta}[T(f(\delta a))-I] x=A(a) x
$$

whenever the limit exists. The domain of $A(a)$ will be denoted by $\mathfrak{D}(a)$; it is clearly a linear subspace of $\mathfrak{X}$.

THEOREM 6.1. Under the assumptions of Theorem 5.1 the set $\mathfrak{D}(a)$ is dense in $\mathfrak{X}$ for each $a$ in $\bar{E}_{n}^{+}$. In particular, $\mathfrak{D}(a)$ contains all elements of the form

$$
\int_{\alpha}^{\beta} T[f(\rho a)] y d \rho, \quad y \in \mathfrak{X}, 0 \leqq \alpha<\beta<\infty .
$$

This is a well known result in the theory of one-parameter semigroups (N. Dunford [2], cf. [6, p. 185]). We also observe that for $x$ in $\mathfrak{D}(a)$ we have

$$
\frac{d}{d \rho} T[f(\rho a)] x=T[f(\rho a)] A(a) x=A(a) T[f(\rho a)] x .
$$

The operator $A(a)$ which is closed is ordinarily unbounded on $\mathfrak{D}(a)$. Its resolvent is given by the Laplace transform

$$
R[\lambda ; A(a)] x=\int_{0}^{\infty} e^{-\lambda \rho} T[f(\rho a)] x d \rho, \quad \Re(\lambda)>0 .
$$

The spectrum of $A(a)$ may very well fill the complementary halfplane $\Re(\lambda) \leqq 0$. $\mathfrak{D}(a)$ is the range of $R[\lambda ; A(a)]$ for any fixed $\lambda$ with $\Re(\lambda)>0$. If $R[\lambda ; A(a)]$ is known, it determines $T[f(\rho a)]$ uniquely with the aid of the inversion formulas for the Laplace transform.

The mapping $a \rightarrow A(a)$ defines a correspondence between the vectors $a$ of $\bar{E}_{n}^{+}$and the infinitesimal generators $A(a)$ of $\mathfrak{S}$. Under suitable assumptions this correspondence is actually an isomorphism under the operations of addition and multiplication by positive numbers. Our previous postulates suffice for the scalar multiplication, however, since (6.1) shows that we have

THEOREM 6.2. Under the assumptions of Theorem 5.1 we have $\mathfrak{D}(\alpha a)=\mathfrak{D}(a)$ for $\alpha>0$ and $A(\alpha a)=\alpha A(a)$.

For the addition and for the fundamental theorems we need further restrictions and the following are convenient assumptions.

$\mathrm{A}_{4}^{3}$. There exists a positive $\rho_{0}$ such that $|a|<\rho_{0},|b|<\rho_{0}, a \neq b$ implies that $T(a) \neq T(b)$. 
$\mathrm{A}_{6}^{4} . F(a, b)$ has continuous partial derivatives with respect to the components of $a$ and $b u p$ to and including the third order.

At every point of $\bar{E}_{n}^{+}$the function $F(a, b)$ may then be expanded in a Taylor series up to terms of the third order. Because of $A_{1}^{4}$ these expansions have a special form: at the origin we have in particular

$$
\begin{aligned}
\gamma_{i} & =\alpha_{i}+\beta_{i}+\sum_{j} \sum_{k} \alpha_{j k}^{i} \alpha_{j} \beta_{k}+R_{3, i}, \quad i=1,2, \cdots, n, \\
a & =\left(\alpha_{i}\right), \quad b=\left(\beta_{i}\right), \quad c=\left(\gamma_{i}\right), \quad c=F(a, b) .
\end{aligned}
$$

The crux of the problem before us is to construct elements of $\mathfrak{X}$ dense in $\mathfrak{X}$ belonging to the domains of definitions of $A(a)$ and of $A(a) A(b)$ for every choice of $a$ and $b$ in $\bar{E}_{n}^{+}$. For this purpose we shall use a modification of artifices due to N. Dunford [2], I. Gelfand [5], and L. Gårding [4]. The same device gives elements belonging to the domain of existence of the product of three or more infinitesimal generators, provided we assume the existence of enough derivatives.

Let $K(c)$ be a numerically-valued function of class $C^{(m)}, m \geqq 3$, defined in $\bar{E}_{n}^{+}$. Let $D$ be a bounded domain whose closure lies in $E_{n}^{+}$ and let $K(c)$ be integrable over $D$ and vanish outside of $D$. If $D_{\eta}$ is a homeomorphic image of $D$ in $E_{n}^{+}$such that no two corresponding points are at a distance of more than $\eta$ apart, then we suppose that

$$
\int|K(c)| d c=o(\eta) \quad \text { when } \eta \rightarrow 0,
$$

where the integration is extended over that part of $D$ which is not in $D_{\eta}$. We require that the partials of order not greater than $m$ have the same integrability properties. The class of all such kernels $K(c)$ will be denoted by $\Omega$. Specifically we may choose $D$ as the cube $0<\sigma$ $<\gamma_{j}<\tau<\infty, j=1, \cdots, n$, and define $K(c)$ in the cube as

$$
\begin{aligned}
K(c) & =K(c ; \sigma, \tau) \\
& =[C(\tau-\sigma)]^{-n} \exp \left\{-(\tau-\sigma)^{2} \sum_{j=1}^{n}\left[\left(\tau-\gamma_{j}\right)\left(\gamma_{j}-\sigma\right)\right]^{-1}\right\}
\end{aligned}
$$

where $C=\int_{0}^{1} \exp \left\{-[\gamma(1-\gamma)]^{-1}\right\} d \gamma$.

For any choice of $K(c)$ in $\Omega$ we define

$$
y=K[x]=\int_{D} K(c) T(c) x d c, \quad x \in \mathfrak{X} .
$$

For fixed $K(c)$ this is a linear bounded transformation of $\mathfrak{X}$ to $\mathfrak{X}$ the 
norm of which does not exceed the integral of $|K(c)|$ over $D$. If $K(c)=K(c ; \sigma, \tau)$ the norm is at most one.

THEOREM 6.3. Under the assumptions $\mathrm{A}^{1}, \mathrm{~A}^{2}, \mathrm{~A}_{3}^{3}$, and $\mathrm{A}_{1}^{4}$ to $\mathrm{A}_{6}^{4}$ the set $\left[\bigcap_{a} \mathfrak{D}(a)\right] \cap\left[\bigcap_{a, b} \mathfrak{D}[A(a) A(b)]\right]$ is dense in $\mathfrak{X}$ and contains the set $\mathfrak{\Omega}[\mathfrak{X}]$ of elements of the form (6.8).

That $\Omega[\mathfrak{X}]$ is dense in $\mathfrak{X}$ follows from

$$
\lim _{\sigma \rightarrow 0} \lim _{\tau \rightarrow \sigma} K(c ; \sigma, \tau)[x]=x
$$

in the sense of strong convergence for every $x$.

We proceed to indicate briefly how one shows that $K[x] \in \mathfrak{D}(a)$ where, without restricting the generality, we may take $|a|=1$. We form

$$
\begin{aligned}
\frac{1}{\delta}\{T[f(\delta a)]-I\} & \\
& =\frac{1}{\delta} \int_{D} K(c)\{T[F(f(\delta a), c)]-T(c)\} x d c
\end{aligned}
$$

In order to find the limit of the right member, we have to study the mapping $U_{\delta}$ defined by $b=F[f(\delta a), c]$ which takes $D$ into a set $D(\delta)$. Condition $\mathrm{A}_{3}^{4}$ asserts that the correspondence is one-to-one for sufficiently small values of $\delta$ since $|f(\delta a)| \leqq B^{-1}\left[e^{B \delta}-1\right]<2 \delta$ if $B \delta<1$. Further

$$
|b-c|<(1+B|c|)|f(\delta a)|<M \delta
$$

where $M$ depends only upon $B$ and $D$. The mapping is consequently a homeomorphism involving only a small distortion of $D$ when $\delta$ is small. It follows that (6.6) holds with $\eta$ replaced by $\delta$ and $D_{\eta}$ by $D(\delta)$.

By $\mathrm{A}_{3}^{4}$ we can solve the equation $b=F[f(\delta a), c]$ for $c$ when $\delta$ is small obtaining the unique solution $c=\psi(b, f(\delta a))$. This solution is continuous by $\mathrm{A}_{3}^{4}$; the added condition $\mathrm{A}_{6}^{4}$ also makes it differentiable. It follows from the theorem on implicit functions that the solution $c=\psi(b, s)$ of the equation $F(s, c)=b$ has continuous partial derivatives with respect to the components of $b$ and $s$ of order not greater than 3 which is the limit for the existence of partials of $F(p, q)$ postulated in $\mathrm{A}_{6}^{\mathbf{4}}$. In this case $f(\delta a)$ has continuous derivatives with respect to $\delta$ of order not greater than 3 as is seen from equation (5.4). It follows that $\psi(b, f(\delta a))$ has continuous partials with respect to the components of $b$ and with respect to $\delta$ of order not greater than 3 . Further, the Jacobian 


$$
J(c ; b)=J[\psi(b, f(\delta a)) ; b]
$$

of the inverse transformation $U_{\delta}^{-1}$ from $D(\delta)$ to $D$ is near to one uniformly in $D$ when $\delta$ is small and has partial derivatives with respect to the components of $b$ and with respect to $\delta$ of order not greater than 2.

From this we conclude that the right member of (6.10) may be written

$$
\begin{aligned}
\delta^{-1} \int_{D(\delta)} K(c) T(b) x J(c ; b) d b-\delta^{-1} \int_{D} K(b) T(b) x d b \\
=\int_{D_{1}} \delta^{-1}[K(c) J(c ; b)-K(b)] T(b) x d b \\
\quad+\delta^{-1} \int_{D_{2}} K(c) J(c ; b) T(b) x d b-\delta^{-1} \int_{D_{3}} K(b) T(b) x d b \\
=J_{1}+J_{2}+J_{3}
\end{aligned}
$$

where $D_{1}=D \cap D(\delta), D_{2}=D(\delta)-D_{1}, D_{3}=D-D_{1}$. Here the norms of $J_{2}$ and $J_{3}$ do not exceed $\|x\|$ times

$$
\delta^{-1} \int_{D_{2}}|K(c)| J(c ; b) d b \text { and } \delta^{-1} \int_{D_{3}}|K(b)| d b
$$

respectively. Both of these expressions tend to zero with $\delta$ by (6.6). It follows that (6.10) tends to a limit so that $y \in \mathfrak{D}(a)$ and

$$
\begin{aligned}
A(a) y & =\int_{D} K_{1}(b ; a) T(b) x d b, \\
K_{1}(b ; a) & =\frac{\partial}{\partial \delta}\left\{K[\psi(b, f(\delta a))] J[\psi(b, f(\delta a) ; b]\}_{\delta=0} .\right.
\end{aligned}
$$

For $D$ near to the origin this becomes

$$
\begin{aligned}
K_{1}(b ; a)=- & K(b)\left[\sum_{i} \sum_{i} \alpha_{j i}^{i} \alpha_{i}+O(|b|)\right] \\
& -\sum_{i} K_{i}(b)\left[\alpha_{i}+\sum_{j} \sum_{k} \alpha_{j k}^{i} \alpha_{j} \beta_{k}+O\left(|b|^{2}\right)\right],
\end{aligned}
$$

where $K_{i}(b)$ is the partial of $K(b)$ with respect to $\beta_{i}$. It should be observed that the remainder terms are independent of the kernel. We conclude that $A(a) K$ is a linear bounded transformation on $\mathfrak{X}$ to $\mathfrak{X}$ and 


$$
\|A(a) K\| \leqq \int_{D}\left|K_{1}(b ; a)\right| d b .
$$

Moreover, it is not difficult to see that this bound is a bounded function of $a$ for $|a|=1$.

We have now to consider the existence of $A\left(a_{1}\right) A\left(a_{2}\right) y$. Here $A\left(a_{2}\right) y$ is given by (6.12) with $a$ replaced by $a_{2}$. This integral is of the same type as (6.8) with a kernel $K_{1}\left(c ; a_{2}\right)$ instead of $K(c)$. Here $K_{1}\left(c ; a_{2}\right)$ has the same properties as $K(c)$ except for differentiability; however, $K_{1}\left(c ; a_{2}\right) \in C^{(2)}$ at least and this more than suffices for our needs. The argument given above may consequently be used also to prove the existence of $A\left(a_{1}\right) A\left(a_{2}\right) y$. Further we see that $A\left(a_{1}\right) A\left(a_{2}\right) K$ is a bounded linear operator and the bound is a bounded function of $a_{1}$ and $a_{2}$ on the unit sphere in $\bar{E}_{n}^{+}$. This completes the proof.

We have seen that the set $\Omega[\mathfrak{X}]$ is dense in $\mathfrak{D}(a)$. Actually a stronger statement can be made and we can make assertions about the graphs of the operators in the relevant product spaces.

TheOREM 6.4. The graph $[y, A(a) y], y \in \Omega[\mathfrak{X}]$, is dense in the graph $[x, A(a) x], x \in \mathfrak{D}(a)$, in $\mathfrak{X} \times \mathfrak{X}$. More generally, the graph $\left[y, A\left(a_{1}\right) y, \cdots, A\left(a_{k}\right) y\right], y \in \Omega[\mathfrak{X}]$, is dense in the graph $\left[x, A\left(a_{1}\right) x\right.$, $\left.\cdots, A\left(a_{k}\right) x\right], x \in \bigcap_{1}^{\mathfrak{k}} \mathfrak{D}\left(a_{j}\right)$, in $\mathfrak{X} \times \cdots \times \mathfrak{X}(k+1$ factors $)$.

The proof is long and laborious so we shall merely sketch the argument for $k=1$ and indicate briefly the extension to more dimensions. Since $A(a)$ is a closed linear operator the graph $\oiint_{1}=[x, A(a) x]$, $x \in \mathfrak{D}(a)$, with points $g_{1}$ may be made into a $(B)$-space under the norm $\left\|g_{1}\right\|=\|x\|+\|A(a) x\|$ with obvious definition of the algebraic operations. If the subset $\mathfrak{S}_{10}=[y, A(a) y], y \in \Omega[\mathfrak{X}]$, is non-dense in $\mathfrak{S}_{1}$, then there exists a linear bounded functional on $\mathscr{S}_{1}$ which vanishes on $S_{10}$ without vanishing identically. Any bounded linear functional on $\mathrm{BS}_{1}$ is of the form

$$
g_{1}^{*}\left(g_{1}\right)=x_{1}^{*}(x)+x_{2}^{*}[A(a) x]
$$

where $x_{1}^{*}$ and $x_{2}^{*}$ are arbitrary linear bounded functionals on $\mathfrak{X}$. We have then for some special choice of $x_{1}^{*}$ and $x_{2}^{*}$ that

$$
x_{1}^{*}(y)+x_{2}^{*}[A(a) y]=0
$$$$
y \in \Omega[\mathfrak{X}],
$$

and by assumption this does not hold for all $x$ in $\mathfrak{D}(a)$ if we replace $y$ by $x$. We now make a special choice of $y$ in $\Omega[\mathfrak{X}]$. We take as domain of integration the cube $C(\delta, \epsilon): \delta<\gamma_{i}<\epsilon, i=1,2, \cdots, n$, and set $K(c)=0$ outside the cube and equal to $\prod_{1}^{n} K_{0}\left(\gamma_{i}\right)$ in the cube where 


$$
K_{0}(\gamma)=\exp \left\{-\eta(\epsilon-\delta)[(\epsilon-\gamma)(\gamma-\delta)]^{-1}\right\}, \quad \eta>0 .
$$

The corresponding elements $y=y(x ; \delta, \epsilon, \eta), x \in \mathfrak{D}(a)$, are in $\mathbb{\Omega}[\mathfrak{X}]$. Substituting this value of $y$ in (6.14) gives an identity in $\delta, \epsilon, \eta$, and $x$. By continuity we may let $\delta \rightarrow 0$ and afterwards $\eta \rightarrow 0$. Under these two operations $x_{1}^{*}(y)$ is carried into

$$
\int_{c(0, \epsilon)} x_{1}^{*}[T(c) x] d c
$$

since $K_{0}(\gamma) \rightarrow 1$ boundedly in $C(0, \epsilon)$. For the discussion of $x_{2}^{*}[A(a) y]$ we use (6.13) which gives the corresponding kernel. This expression involves two terms of which the first one leads to

$$
\int_{C(0, \epsilon)}\left\{\sum_{i} \sum_{j} \alpha_{j i}^{i} \alpha_{j}+O(|c|)\right\} x_{2}^{*}[T(c) x] d c .
$$

We recall that the remainder term is independent of the kernel. The second term of (6.13) gives rise to $n$ singular integrals of which the first one, $i=1$, involves the integral

$$
\eta \int_{0}^{\epsilon} K_{0}\left(\gamma_{1}\right)\left[\left(\gamma_{1}-\epsilon\right)^{-2}-\gamma_{1}^{-2}\right] f_{1}(c) d \gamma_{1}
$$

which is multiplied by $\prod_{2}^{n} K_{0}\left(\gamma_{j}\right)$ integrated with respect to the remaining $n-1$ variables from 0 to $\epsilon$. Here

$$
f_{i}(c)=\left[\alpha_{i}+\sum_{j} \sum_{k} \alpha_{j k}^{i} \alpha_{j} \gamma_{k}+O\left(|c|^{2}\right)\right] x_{2}^{*}[T(c) x] .
$$

Passing to the limit with $\eta$ gives the result

$$
f_{1}\left(\epsilon, \gamma_{2}, \cdots, \gamma_{n}\right)-f_{1}\left(0, \gamma_{2}, \cdots, \gamma_{n}\right)
$$

as is easily seen. At the same time $\prod_{2}^{n} K_{0}\left(\gamma_{j}\right) \rightarrow 1$. Thus we see that the limit of $x_{2}^{*}[A(a) y]$ under the limit processes $\delta \rightarrow 0, \eta \rightarrow 0$ becomes the integral over the $(n-1)$-dimensional boundary of the cube $C(0, \epsilon)$ of the function which on the face $\gamma_{i}=\epsilon$ equals $f_{i}(c)$ and on the face $\gamma_{i}=0$ equals $-f_{i}(c)$.

After performing these operations on $x_{1}^{*}(y)+x_{2}^{*}[A(a) y]$ we multiply by $\epsilon^{-n}$ and let $\epsilon \rightarrow 0$. The contributions from (6.15) and (6.16) add up to

$$
x_{1}^{*}(x)-\left\{\sum_{i} \sum_{i} \alpha_{j i}^{i} \alpha_{j}\right\} x_{2}^{*}(x) .
$$

The surface integral leads to two terms of which one arises from the 
partials of the factors $\alpha_{i}+\sum_{j} \sum_{k} \alpha_{j k}^{\alpha} \alpha_{j} \gamma_{k}+O\left(|c|^{2}\right)$ and gives a limit which cancels the second term of (6.17). The second term involves combinations of difference quotients of $x_{2}^{*}[T(c) x]$. Since everything else tends to a limit when $\epsilon \rightarrow 0$, this term must also tend to a limit which we denote by $X^{*}(x)$. We have then

$$
x_{1}^{*}(x)+X^{*}(x)=0,
$$

$x \in \mathfrak{D}(a)$.

Since $\mathfrak{D}(a)$ is dense in $\mathfrak{X}$, this must hold for all $x$ so that $X^{*}=-x_{1}^{*}$. But for $x=y$ in $\Omega[\mathfrak{X}]$ we have $X^{*}(y)=x_{2}^{*}[A(a) y]$ and $\Omega[\mathfrak{X}]$ is dense in $\mathfrak{D}(a)$ so we must have $X^{*}(x)=x_{2}^{*}[A(a) x]$ for all $x$ in $\mathfrak{D}(a)$. But this shows that (6.14) holds with $y$ replaced by any $x$ of $\mathfrak{D}(a)$ so that $g_{1}^{*}\left(g_{1}\right)=0$ for all $g_{1}$. Thus $\mathfrak{B}_{10}$ is dense in $\mathbb{S}_{1}$.

The extension from 1 to $k$ does not offer any new difficulties. The graph $\mathbb{S}_{k}=\left[x, A\left(a_{1}\right) x, \cdots, A\left(a_{k}\right) x\right], x \in \bigcap_{1}^{k} \mathfrak{D}\left(a_{j}\right)$, becomes a $(B)$ space under the norm $\left\|g_{k}\right\|=\|x\|+\sum_{1}^{k}\left\|A\left(a_{j}\right) x\right\|$ and the linear functionals on $\mathbb{S}_{k}$ are of the form

$$
g_{k}^{*}\left(g_{k}\right)=x_{1}^{*}(x)+x_{2}^{*}\left[A\left(a_{1}\right) x\right]+\cdots+x_{k+1}^{*}\left[A\left(a_{k}\right) x\right] .
$$

Proceeding as above with the same choice of $y=y(x ; \delta, \epsilon, \eta), x$ $\in \bigcap_{1}^{k} \mathfrak{D}\left(a_{j}\right)$, and passing to the limit with the parameters, one obtains a relation of type (6.18) holding for all $x$ under consideration. But for $x=y \in \Omega[\mathfrak{X}]$ we have

$$
X^{*}(y)=x_{2}^{*}\left[A\left(a_{1}\right) y\right]+\cdots+x_{k+1}^{*}\left[A\left(a_{k}\right) y\right] .
$$

Here $X^{*}$ has a unique extension when we pass from the dense set $\Re[\mathfrak{X}]$ to the set $\bigcap_{1}^{k} \mathfrak{D}\left(a_{j}\right)$ and the right-hand side of (6.19) has the obvious extension obtained by replacing $y$ by $x$. It follows that (6.19) holds for all points of $\bigcap_{1}^{k} \mathfrak{D}\left(a_{j}\right)$ so that $g_{k}^{*}$ is the zero functional and the subgraph is dense in $\mathbb{S}_{k}$.

We now define an operator $U(a)$ by

$$
U(a) x=\lim _{\delta \rightarrow 0} \delta^{-1}[T(\delta a)-I] x
$$

whenever the limit exists.

Theorem 6.5. $U(a) y$ exists and equals $A(a) y$ if $y \in \Omega[\mathfrak{X}]$.

The existence of $U(a) y$ is proved by the argument used in the proof of Theorem 6.3. We have merely to replace $f(\delta a)$ by $\delta a$ throughout. But $f(\delta a)-\delta a=O\left(\delta^{2}\right)$ whence it follows that

$$
\begin{gathered}
\psi(b, f(\delta a))-\psi(b, \delta a)=O\left(\delta^{2}\right), \\
J[\psi(b, f(\delta a)) ; b]-J[\psi(b, \delta a) ; b]=O\left(\delta^{2}\right) .
\end{gathered}
$$


so the right member of (6.13) is unchanged when we replace $f(\delta a)$ by $\delta a$. Hence $U(a) y=A(a) y$ for $y \in \Omega[\mathfrak{X}]$. If it could be shown that $U(a)$ is a closed operator, then it would follow from Theorem 6.4 that the domain of $U(a)$ contains that of $A(a)$ and that equality between the operators holds in $\mathfrak{D}(a)$. At present we cannot decide this question and it is not vital for the following discussion.

THEOREM 6.6. If $y=K(c)[x]$ and if $a_{1}, a_{2}, b$ are points of $\bar{E}_{n}^{+}$with $\left|a_{1}\right|<\delta(D),\left|a_{2}\right|<\delta(D),|b| \leqq 1$, then there exists a constant $C$ depending only upon $K(c)$ such that

$$
\left|\left[T\left(a_{1}\right)-T\left(a_{2}\right)\right] y\right| \leqq C\left|a_{1}-a_{2}\right|\|x\|
$$

and the same relation holds with $y$ replaced by $A(b) y$.

This is proved by the method of Theorem 6.3. We omit the details.

We can now prove that the correspondence $a \rightarrow A(a)$ is a homomorphism under addition in the following sense.

THEOREM 6.7. $\mathfrak{D}\left(a_{1}+a_{2}\right)$ contains $\mathfrak{D}\left(a_{1}\right) \cap \mathfrak{D}\left(a_{2}\right)$ and in the latter set

$$
A\left(a_{1}+a_{2}\right) x=A\left(a_{1}\right) x+A\left(a_{2}\right) x .
$$

We start by proving (6.22) for $x=y \in \Omega[\mathfrak{X}]$. In this set it is sufficient to prove the corresponding relation with $A$ replaced by $U$ and this is accomplished if we can show that

$$
\left[T\left(\delta\left(a_{1}+a_{2}\right)\right)-T\left(\delta a_{1}\right)-T\left(\delta a_{2}\right)+I\right] y=o(\delta)
$$

when $\delta \rightarrow 0$. The left member equals

$$
\left\{T\left(\delta\left(a_{1}+a_{2}\right)\right)-T\left[F\left(\delta a_{1}, \delta a_{2}\right)\right]\right\} y+\left[T\left(\delta a_{1}\right)-I\right]\left[T\left(\delta a_{2}\right)-I\right] y .
$$

By Theorem 6.6 the norm of the first term does not exceed

$$
C\left|\delta\left(a_{1}+a_{2}\right)-F\left(\delta a_{1}, \delta a_{2}\right)\right|\|x\|
$$

which is $O\left(\delta^{2}\right)$ by $A_{5}^{4}$ since $\omega(\xi)=O(\xi)$. Since $y \in \mathcal{D}\left(a_{2}\right)$, we have $\left[T\left(\delta a_{2}\right)-I\right] y=\delta A\left(a_{2}\right) y+o(\delta)$, so that

$$
\begin{aligned}
\left\|\left[T\left(\delta a_{1}\right)-I\right]\left[T\left(\delta a_{2}\right)-I\right] y\right\| \leqq \delta\left\|\left[T\left(\delta a_{1}\right)-I\right] A\left(a_{2}\right) y\right\| \\
+o(\delta)\left\|T\left(\delta a_{1}\right)-I\right\| \leqq C \delta^{2}\left|a_{1}\right|\|x\|+o(\delta)
\end{aligned}
$$

from which the assertion follows. In order to extend the validity of (6.22) from $\Omega[\mathfrak{X}]$ to $\mathfrak{D}\left(a_{1}\right) \cap \mathfrak{D}\left(a_{2}\right)$ we argue as follows. By Theorem 6.4 the subgraph $\left[y, A\left(a_{1}\right) y, A\left(a_{2}\right) y\right], y \in \Omega[\mathfrak{X}]$, is dense in the graph $\left[x, A\left(a_{1}\right) x, A\left(a_{2}\right) x\right], x \in \mathfrak{D}\left(a_{1}\right) \cap \mathfrak{D}\left(a_{2}\right)$. Hence for any $x$ in the latter set we may find a sequence $y_{n} \in \Omega[\mathfrak{X}]$ such that $y_{n} \rightarrow x, A\left(a_{1}\right) y_{n}$ $\rightarrow A\left(a_{1}\right) x, A\left(a_{2}\right) y_{n} \rightarrow A\left(a_{1}\right) x$. It follows that 


$$
A\left(a_{1}+a_{2}\right) y_{n}=A\left(a_{1}\right) y_{n}+A\left(a_{2}\right) y_{n} \rightarrow A\left(a_{1}\right) x+A\left(a_{2}\right) x .
$$

Since $A\left(a_{1}+a_{2}\right)$ is also a closed operator, it follows that $A\left(a_{1}+a_{2}\right) x$ exists and that (6.22) holds.

We come now to the main theorem of this section.

THEOREM 6.8. Let

$$
a=\left(\alpha_{1}, \alpha_{2}, \cdots, \alpha_{n}\right)=\alpha_{1} e_{1}+\alpha_{2} e_{2}+\cdots+\alpha_{n} e_{n}
$$

and set $A\left(e_{k}\right)=A_{k}$. Then $\mathfrak{D}(a)$ contains the set $\bigcap_{1}^{n} \mathfrak{D}\left(e_{k}\right) \equiv \mathfrak{D}_{n}$ and for $x$ in the latter set

$$
A(a) x=\alpha_{1} A_{1} x+\alpha_{2} A_{2} x+\cdots+\alpha_{n} A_{n} x .
$$

The basic infinitesimal generators $A_{1}, A_{2}, \cdots, A_{n}$ are linearly independent in $\mathfrak{D}_{n}$.

The validity of (6.23) is an immediate consequence of the preceding theorem. A linear relation with constant coefficients between $A_{1} x, A_{2} x, \cdots, A_{n} x$ valid for all $x$ in $\mathfrak{D}_{n}$ implies the existence of two distinct vectors $a$ and $b$ in $\bar{E}_{n}^{+}$such that $A(a) x=A(b) x$ in $\mathfrak{D}_{n}$. We know that the subgraph $[y, A(a) y], y \in \Omega[\mathfrak{X}]$, is dense in the graph of $A(a)$. A fortiori this is true for the graph $[x, A(a) x], x \in \mathfrak{D}_{n}$. Now for $\Re(\lambda)>0$ the set $[\lambda I-A(a)][\mathfrak{D}(a)]$ is dense in $\mathfrak{X}$ so the same must be true for $[\lambda I-A(a)]\left[\mathfrak{D}_{n}\right]=[\lambda I-A(b)]\left[\mathfrak{D}_{n}\right]$. From this we conclude that the linear bounded operators $R[\lambda ; A(a)]$ and $R[\lambda ; A(b)]$ coincide in a dense set and hence everywhere. By the inversion formulas for the Laplace transform, applied to formula (6.4), we conclude that the corresponding one-parameter semi-group operators $T[f(\rho a)]$ and $T[f(\rho b)]$ are identical for all values of $\rho$. But for all small values of $\rho$ we have $f(\rho a) \neq f(\rho b)$ and $|f(\rho a)|<\rho_{0},|f(\rho b)|<\rho_{0}$. Thus the result contradicts $\mathrm{A}_{4}^{3}$ so we conclude that $A(a)$ and $A(b)$ are distinct operators on $\mathfrak{D}_{n}$ when $a \neq b$.

The infinitesimal generators $A(a)$ of $\subseteq$ form an $n$-dimensional system which is closed under addition and multiplication by positive numbers. On the other hand, multiplication by negative numbers is not allowed since $a$ and $-a$ are not simultaneously in $\bar{E}_{n}$ if $a \neq 0$. In this respect there is a striking difference between the semi-module of infinitesimal generators of a semi-group and the Lie ring of generators of a group.

7. The fundamental theorems. We come now to the analogues of the three fundamental theorems of Lie.

TheOREM 7.1. For $y \in \Omega[\mathfrak{X}]$ and small values of $a$ in $E_{n}^{+}$ 


$$
\frac{\partial}{\partial \alpha_{j}} T(a) y=\sum_{k=1}^{n} \Gamma_{j k}(a) T(a) A_{k} y, \quad j=1,2, \cdots, n,
$$

where the matrix $\left(\Gamma_{j k}(a)\right)$ tends to the unit matrix when $a \rightarrow 0$. If the operator in the left member is closed, then its domain contains $\mathfrak{D}_{n}$ and (7.1) holds in $\mathfrak{D}_{n}$.

The left side of (7.1) is the limit when $\delta \rightarrow 0$ of

$$
\delta^{-1}\left[T\left(a+\delta e_{j}\right)-T(a)\right] y .
$$

Using $\mathrm{A}_{\mathbf{3}}^{\mathbf{4}}$ we see that the equation $F(a, h)=a+\delta e_{j}$ has a unique solution in $\bar{E}_{n}^{+}$, viz.

$$
h=\psi\left(a+\delta e_{j}, a\right)=\chi_{j}(a, \delta)=c_{j}(a) \delta+O\left(\delta^{2}\right),
$$

where the components $\Gamma_{j k}(a)$ of the vector $c_{j}(a)$ are determined by the linear system of equations

$$
\sum_{k=1}^{n} F_{i k}(a, 0) \Gamma_{j k}(a)=\delta_{i j}, \quad i=1,2, \cdots, n .
$$

Here $F_{i k}$ as in formula (5.4) is the partial derivative of the $i$ th component of $F(a, b)$ with respect to the $k$ th component of $b$ in which we set $b=0$. Since $F_{i k}(a, 0) \rightarrow \delta_{i k}$ when $|a| \rightarrow 0$, the determinant of the system (7.3) is different from zero for small values of $|a|$ so that the $\Gamma_{j k}(a)$ may be determined. Further $\Gamma_{j k}(a) \rightarrow \delta_{j k}$ when $|a| \rightarrow 0$. Thus

$$
\begin{aligned}
\delta^{-1}\left[T\left(a+\delta e_{j}\right)-T(a)\right] & y \\
= & T(a) \delta^{-1}\left\{T\left[\chi_{j}(a, \delta)\right]-I\right\} y \\
= & T(a) \delta^{-1}\left\{T\left[\delta c_{j}(a)\right]-I\right\} y \\
& +T(a) \delta^{-1}\left\{T\left[\chi_{j}(a, \delta)\right]-T\left[\delta c_{j}(a)\right]\right\} y .
\end{aligned}
$$

The first term in the last member tends to $T(a) A\left[c_{j}(a)\right] y$ when $\delta \rightarrow 0$ by Theorem 6.5 while the second term tends to $\theta$ by Theorem 6.6. Hence the limit exists and by Theorem 6.8 it equals

$$
T(a) A\left[c_{j}(a)\right] y=\sum_{k=1}^{n} \Gamma_{j k}(a) A_{k} y .
$$

The left side of this equation involves a closed operator, but it is not a priori obvious that the partials of $T(a)$ are closed operators; if they are, then Theorem 6.4 shows immediately that (7.1) holds in $\mathfrak{D}_{n}$.

Theorem 7.1 also gives us

$$
T(a) A_{i} y=\sum_{k=1}^{n} \Delta_{i k}(a) \frac{\partial}{\partial \alpha_{k}} T(a) y
$$


where $\left(\Delta_{i k}(a)\right)$ is the inverse of the matrix $\left(\Gamma_{j k}(a)\right)$. It follows from (7.3) that

$$
\Delta_{i k}(a)=F_{k i}(a, 0) .
$$

The second and third fundamental theorems involve the structural constants of the semi-group defined by

$$
\gamma_{j k}^{i}=\alpha_{j k}^{i}-\alpha_{k i}^{i}, \quad \alpha_{j k}^{i}=\left(\frac{\partial^{2} F_{i}}{\partial \alpha_{j} \partial \beta_{k}}\right)_{0,0}
$$

in the notation of (6.5). We note that

$$
\frac{\partial}{\partial \alpha_{i}} \Delta_{i k}(a) \rightarrow \alpha_{j i}^{k}, \quad|a| \rightarrow 0 .
$$

Theorem 7.2. If $y \in \Omega[\mathfrak{X}]$

$$
\left[A_{i}, A_{j}\right] y \equiv\left(A_{i} A_{j}-A_{j} A_{i}\right) y=\sum_{m=1}^{n} \gamma_{i j}^{m} A_{m} y .
$$

If the operator in the first member is closed, then the relation holds in $\mathfrak{D}_{n}$.

For the proof we use (7.5) twice forming

$$
\begin{aligned}
T(a) A_{i} A_{j} y & =\sum_{k=1}^{n} \Delta_{i k}(a) \frac{\partial}{\partial \alpha_{k}}\left[T(a) A_{i} y\right] \\
& =\sum_{k=1}^{n} \Delta_{i k}(a) \frac{\partial}{\partial \alpha_{k}}\left\{\sum_{m=1}^{n} \Delta_{j m}(a) \frac{\partial}{\partial \alpha_{m}} T(a) y\right\} .
\end{aligned}
$$

Interchanging $i$ and $j$, subtracting and simplifying we get

$$
T(a)\left[A_{i}, A_{j}\right] y=\sum_{m=1}^{n} \Delta_{i j}^{m}(a) \frac{\partial}{\partial \alpha_{m}} T(a) y
$$

where

$$
\Delta_{i j}^{m}(a)=\sum_{k=1}^{n}\left\{\Delta_{i k}(a) \frac{\partial}{\partial \alpha_{k}} \Delta_{j m}(a)-\Delta_{j k}(a) \frac{\partial}{\partial \alpha_{k}} \Delta_{i m}(a)\right\},
$$

which tends to $\gamma_{i j}^{m}$ when $|a| \rightarrow 0$ by (7.8) since $\Delta_{i k}(a) \rightarrow \delta_{i k}$. Formula (7.1) shows that the partial of $T(a) y$ with respect to $\alpha_{m}$ tends to $A_{m} y$. It should be observed that the second order partials of $T(a) y$ which arise in the process, but cancel in the subtraction, actually exist when $y \in \mathfrak{R}[\mathfrak{X}]$. This follows from the fact that in (7.4) we may replace $y$ by $A_{i} y$ and still carry through the limit process. The second partials may consequently be found by formal differentiation of 
formula (7.1). If $\left[A_{i}, A_{j}\right]$ is a closed operator, then Theorem 6.4 shows that (7.9) holds in $\mathfrak{D}_{n}$.

The second fundamental theorem asserts that each commutator $\left[A_{i}, A_{j}\right]$ is a linear combination of basic infinitesimal generators, but in the semi-group case it does not follow that $\left[A_{i}, A_{j}\right]$ is also an infinitesimal generator, that is, we cannot always find a vector $c$ in $\bar{E}_{n}^{+}$such that $\left[A_{i}, A_{j}\right] y=A(c) y$. In particular, we note that if $\left[A_{i}, A_{j}\right]$ is an infinitesimal generator, $\left[A_{j}, A_{i}\right]$ cannot be one.

THEOREM 7.3. The structural constants satisfy

$$
\begin{gathered}
\gamma_{j k}^{i}=-\gamma_{k j}^{i}, \\
\sum_{m=1}^{n}\left[\gamma_{i m}^{p} \gamma_{j k}^{m}+\gamma_{j m}^{p} \gamma_{k i}^{m}+\gamma_{k m}^{p} \gamma_{i j}^{m}\right]=0 .
\end{gathered}
$$

Here (7.10) follows from (7.7) while (7.11) follows from the relation

$$
\left[A_{i},\left[A_{j}, A_{k}\right]\right]+\left[A_{i},\left[A_{k}, A_{i}\right]\right]+\left[A_{k},\left[A_{i}, A_{i}\right]\right]=\Theta
$$

which holds when the operator on the left acts on the subspace $\mathfrak{\Omega}[\mathfrak{X}]$.

8. Conclusions. The preceding theory raises perhaps more questions than it answers. Let us list some directions in which further research is desirable.

(1) Determine the set of points $c$ such that $c=f(a)$ for some $a$. In particular, are all finite boundary points of this set "accessible" and found by solving the equation $F(p, p)=p$ ?

(2) Extend the investigation to other parameter sets.

(3) Prove that the partials of $T(a)$ and the commutators are closed operators.

(4) Formulate and prove converses of the fundamental theorems.

(5) Is it possible to embed the given semi-group $\mathbb{S}$ in a group of, in general, unbounded operators, the group being generated by the set $\sum \alpha_{k} A_{k}$ with real $\alpha$ 's not necessarily positive?

\section{REFERENCES} 61-101.

1. G. Birkhoff, Analytical groups, Trans. Amer. Math. Soc. vol. 43 (1938) pp.

2. N. Dunford, On one parameter groups of linear transformations, Ann. of Math. (2) vol. 39 (1938) pp. 569-573.

3. N. Dunford and I. E. Segal, Semi-groups of operators and the Weierstrass theorem, Bull. Amer. Math. Soc. vol. 52 (1946) pp. 911-914.

4. L. Gårding, Note on continuous representations of Lie groups, Proc. Nat. Acad. Sci. U. S. A. vol. 33 (1947) pp. 331-332. 
5. I. Gelfand, On one-parametrical groups of operators in a normed space, C. R. (Doklady) Acad. Sci. URSS. N.S. vol. 25 (1939) pp. 713-718.

6. E. Hille, Functional analysis and semi-groups, Amer. Math. Soc. Colloquium Publications, vol. 30 , New York, 1948 , xii +528 pp.

7. I. E. Segal, Topological groups in which multiplication on one side is differentiable, Bull. Amer. Math. Soc. vol. 52 (1946) pp. 481-487.

8. P. A. Smith, Foundations of the theory of Lie groups with real parameters, Ann. of Math. (2) vol. 44 (1943) pp. 481-513.

9. - Foundations of Lie groups, Ann. of Math. (2) vol. 48 (1947) pp. 29-42.

10. $\mathrm{K}$. Yosida, On the differentiability and the representation of one-parameter semigroup of linear operators, Journal of the Mathematical Society of Japan vol. 1 (1948) pp. 15-21.

11. - An operator-theoretical treatment of temporally homogeneous Markoff process, Journal of the Mathematical Society of Japan.

12. - Brownian motion on the surface of the 3-sphere, Ann. Math. Statist. vol. 20 (1949) pp. 292-296.

YALE UNIVERSITY 